\title{
Research on the Teaching Scheme for Postgraduate Courses of Advanced Manufacturing System
}

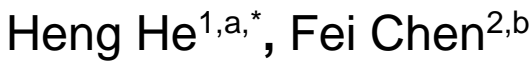 \\ ${ }^{1}$ College of Economics and Management, Guangxi University of Science and Technology, Liuzhou, \\ Guangxi, China \\ ${ }^{2}$ College of Economics and Management, Guangxi University of Science and Technology, Liuzhou, \\ Guangxi, China \\ aheheng0772@163.com, bchenfei_7375@163.com \\ ${ }^{*}$ Corresponding author
}

Keywords: Advanced Manufacturing System, postgraduate, Flipping Classroom, Seminar Teaching.

\begin{abstract}
There are some problems in the previous teaching of Advanced Manufacturing Systems for graduate students, such as the fuzzy orientation of teaching, the "unadvanced" in the course content, and the inadaptability of teaching methods to the characteristics of graduate students' learning, which make the course teaching unable to meet the needs of training innovative talents. With problem analysis, it is proposed that the teaching mode of combining the flipping classroom with the seminar teaching should be adopted in the postgraduate course. furthermore, the teaching scheme of the course is presented. The details in the implementation of the teaching scheme are given from aspects of the teaching materials, the allocation of class hours, the content of teaching, the topics of seminar and the way of assessment. Since the implementation of the scheme for more than one year, good teaching effects have been achieved.
\end{abstract}

\section{先进制造系统研究生课程教学方案的研究

\author{
何恒 ${ }^{1, a,{ }^{*},}$, 陈飞 $2, \mathrm{~b}$ \\ 1 广西科技大学经济与管理学院, 柳州, 广西, 中国 \\ 2 广西科技大学经济与管理学院, 柳州, 广西, 中国 \\ aheheng0772@163.com, bchenfei_7375@163.com
} \\ 通讯作者}

关键词：先进制造系统; 研究生; 翻转课堂; 研讨式教学

中文摘要. 原有的先进制造系统研究生课程教学存在教学定位不清晰, 课程内容不 “先进”, 和教学方式不适应研究生学习特点等问题, 使得课程教学无法满足培养创新型人才的需求。 本文在分析问题的基础上, 提出了该课程应当采取翻转课堂与研讨式教学相结合的教学模式, 由此提出了该课程的教学方案, 从教材来源、课时安排、教学内容、研讨主题的选取和考核 方式等方面给出了教学方案的实施要点。方案实施一年多以来, 取得了良好的教学效果。 


\section{1. 引言}

我校硕士研究生工业工程专业自2015年开始招生。在2016年版的工业工程硕士研究生人 才培养方案中, 先进制造系统被列为专业必修课程。设立这门课程的目的是: 通过课程学习, 全面了解制造系统的现状与发展趋势, 掌握先进制造模式、先进的设计技术、先进的制造工 艺及设备、先进的制造理念和典型的制造系统，从而拓宽学科视野，培养创新意识。

笔者在该课程的教学实践当中遇到了许多问题。特别是, 此前我校一直开设有该课的本 科生课程, 该课程的研究生课程与本科生课程究竟有何差异? 基于对这个问题的思考, 通过 总结以往的教学经验, 逐步形成了一套行之有效的先进制造系统研究生课程的教学方案。

\section{2. 先进制造系统研究生教学存在的问题}

通过总结近几年先进制造系统课程的教学经验，可以归纳出以下问题。

\section{1 研究生与本科生教学定位不清}

2016年版的工业工程硕士研究生人才培养方案执行以前, 先进制造系统是作为专业选修 课开设的。其目的是给那些本科时没有修读该课程的研究生进行“补课”。那时, 该课程的研 究生教学和本科生教学是等同的, 甚至允许研究生跟班修读本科生的课程。

在2016年版的工业工程硕士研究生人才培养方案执行以后, 先进制造系统作为研究生专 业必修课程，仍然延续以往的课程定位显然是不合适的。

\section{2 课程内容无法保持“先进”}

目前该课程的教材采用戴庆辉主编的《先进制造系统》（2010年版）。该书出版距今已 有9年，书本中有些数据已经过时，有些技术已不再“先进”。例如课本中我国2002年第二产业 比重为 $51.8 \%$ ，第三产业为 $33.7 \%$ ，第二产业远高于第三产业; 但是早在 2013 年，我国第三产 业的比重就已经超越了第二产业; 课本中制造业信息化的基础仍然是互联网技术，但现在物 联网、移动互联网、大数据、云计算和人工智能技术正在推动着制造业走向工业4.0时代。如 何使得教学内容与时俱进，保持知识的“先进性”，是该课程教学研究的重要论题。

\section{3 教学方式无法适应研究生的学习特点}

参照文献[1]的分类，可以将我们以往的研究生教学方式归为两种类型：

一、灌输式。老师逐一讲授需要学生掌握的知识点。

二、放养式。老师只管考核，学生自行学习。

灌输式教学压抑了研究学习的积极性和主动性, 无法实现培养创新型人才的目的。而放 养式教学则矫枉过正, 过分强调了学生的自主性, 而缺乏对学习的有效的管理和引导, 同样 不利于研究生培养目标的达成。

\section{3. 先进制造系统研究生教学问题分析}

\section{1 研究生与本科生教学定位}

本科生教育的目标是为社会提供具备基本科研或技术应用能力的人才; 而研究生教育的 目标是为社会提供具有独立从事科研能力的高层次人才 ${ }^{[2]}$ 。因此相比本科生教育, 研究生教 育更加注重创新能力的培养。课程教学是研究生创新能力培养的切入点和关键环节 ${ }^{[3]}$ 。

对于先进制造系统这门课而言, 研究生不仅要像本科生一样掌握基本的知识结构、先进 理念与方法, 更重要的是要把握先进制造系统的学术前沿和发展趋势。 


\section{2 课程内容的动态性特点}

先进制造系统是一门动态性的课程，随着新科技的应用和新理念的出现而不断地更新、 充实和发展，但教材更新的步伐远跟不上技术的发展 ${ }^{[4]}$ 。这也导致课本内容的不“先进”。

由于课程内容的动态性, 研究生教学不应该拘泥于课本。当然, 现行的课本为我们提供 了一个结构完整知识体系, 因此课程的教学还应当以课本为纲。应当根据研究生的教学定位 来合理安排补充教材, 及时更新和完善本课程的知识体系。另外, 本科生课程与研究生课程 的应该在教学内容上关联与统一。前者为后者发展的基础。后者为前者不断开拓新的内容。

\section{3 研究生的学习特点和方式}

当代研究生接受新事物的能力强, 但学习当中也面临知识爆炸、研究课题的多学科性和 专业性的多重挑战。传统的教学方式已经难以满足研究生的学习需求。文[5]归纳研究生的学 习需求为：个性化的学习与互动; 与时俱进的教学内容与资源; 和更多的实训与实践。

近年来翻转课堂教学模式开始被教育界所重视和接受。翻转课堂是指重新调整课内课外 时间, 将学习的决定权从教师转移给学生。在这种教学模式下, 教师不再占用课内时间来讲 授书本知识, 而是要求学生在课前完成自主学习; 而课内时间里学生能更专注于基于项目的 学习, 通过师生互动, 运用所学知识来解决真正感兴趣的问题, 从而获得对知识更深层次的 理解。文献[6,7]指出翻转课堂是一种符合研究生的学习特点的教学模式。

在课堂教学的组织方面, 研讨式教学也在研究生教学中得到了广泛的应用 ${ }^{[8,9]}$ 。该方法是 指师生共同对某研讨主题开展研究与探讨。笔者认为研讨式教学可以分为问题型研讨和专题 型研讨两类。前者以解决问题为目的，而后者则以在某个领域拓展知识为目的。

研讨式教学与翻转课堂教学模式是紧密相关的。研讨是翻转课堂的课内教学的主要组织 形式; 而只有通过翻转课堂的课外学习才能掌握研讨教学所需要的基础知识。在研究生课程 教学实践当中二者可以完美地结合。

\section{4. 先进制造系统研究生课程的教学方案}

根据以上分析, 在研究生课程的教学方案中采用翻转课堂+研讨式教学的模式。具体如下:

\section{1 教材的来源}

核心教材依然选用经典的《先进制造系统》。辅助教材包括《先进制造技术》等相关书 籍。但是, 更多的知识则来源于网络。知识来源可以是科技新闻版块、知名的学术刊物、制 造工程实验室等。同时该课程的教材是可以“生长”的。依托研讨式教学, 建立了网络课件和 专题资料库。上届学生研讨报告经过整理后, 就形成了后来学生的学习资源。

\section{2 课时安排和课程内容}

该课程的课时为 32 个学时, 分为理论教学 (26学时) 和实践教学 (6学时) 两个阶段。

理论教学的内容按照教材的章节进行安排。虽然课程结构一样, 但教学的具体内容是不 同的。按照翻转课堂的教学模式, 学生在课外自学课本上的基础知识。课堂教学的组织方式 为专题型研讨。在每一个章节, 老师事先指定若干个相关的前沿专题供学生选择。学生选题 后在自行收集资料, 撰写专题报告。每次课为 3 个学时, 课堂上老师与同学对各自的选题展开 研讨。

实践教学为6学时, 两次课。组织方式为问题型研讨。即每个学生自行观察一个本地的制 造系统或服务系统，发现其存在的问题，并且运用先进制造系统的基本方法和原理来解决该 问题。第一次课为开题, 即对选题的价值和研究可行性进行评估。第二课次为答辩。开题与 答辩之间应间隔一周以上, 以便学生有足够的时间开展研究。 


\section{3 专题型研讨的选题}

在研讨课开始前一周，老师会指定若干个研讨专题。选题可以是相关领域的最新进展或 研究热点。例如在第一章先进制造系统总论指定了两个研讨专题: 1) 工业4.0与中国制造 2025; 2）生产性服务业的发展现状。可以是推动制造系统重大变革的关键技术。如: 移动互联网对 制造业发展的影响。可以是对社会发展产生重大影响的制造技术。如：新能源汽车的发展现 状。也可以是与当前广泛关注的热点事件相关的技术问题。例如在中美贸易争端背景下提出 的研讨专题：1）5G通讯技术会给制造业发展带来哪些变革？2）剖析华为手机的制造供应链。

\section{4 课程的考核}

课程考核的总成绩由平时成绩（占50\%）和期末成绩（占50\%）构成。平时成绩评分依 据为理论教学阶段学生所作的专题型研讨报告。从中选择 5 篇报告, 每篇报告满分 10 分。期末 成绩的评分依据为实践教学阶段学生所作的问题型研讨报告。

\section{5. 结束语}

新的教学方案实施一年多以来, 取得了良好的效果, 学生们普遍认为: 研究生课程与本 科生课程真的不一样，研究生课程要有趣得多。

通过实施研讨式教学，不仅学生的科研能力得到了增强，教师也在研讨中拓宽了视野， 提高了自身的教学水平。所积累的研讨报告也充实了本科生的教学资料, 对本科生教学水平 的提高起到积极的作用。

\section{致谢}

本文为广西科技大学研究生核心课程建设项目（先进制造系统）的阶段性成果之一。

\section{References}

[1] J.G. Yao, G.F. Pang, S.Q. Huang, J.S. Xie, Knowledge map analysis of graduate management mode, Research and Practice of Medical Education, vol.25, pp. 545-549, 2017.

[2] S.M. Bao, J. Gao, Explore the essence of postgraduate education from comparisons between undergraduate and graduate training programs, Modern Educational Science (Higher Education Research), vol., pp.139-142+145, 2006.

[3] Y.C. Zhang, Postgraduate course teaching under innovative talents training mode, Educational and Teaching Forum,vol. , pp.27-29, 2012.

[4] X.S. Liu, J. Yao, Research and practice on teaching reform of advanced manufacturing systems, Education Exploration, vol., pp.62-63, 2010.

[5] W.Y Yao, Y.J Shen, Y.D. Shen, Internet development and postgraduate teaching reform, Jiangsu Higher Education, vol.213, pp. 79-82, 2018.

[6] F.Q. Wang, L.L. Xuan, "Flipping classroom" and the reform of postgraduate teaching model in universities, Heilongjiang Researches on Higher Education, vol., pp.13-13, 2017.

[7] Z.Y. Cui, R. Zhao, An analysis of postgraduate teaching mode based on internet information technology. Theory and Practice of Education, vol., pp.8-10, 2017

[8] G.L. Geng, Attempts and reflections on the problem-based teaching method in postgraduate education. Educational and Teaching Forum, vol., pp.157-159, 2013. 
[9] J.W. Chen, J. Liu, Teaching postgraduate courses by simulating seminar, Journal of Higher Education Research, vol.31, pp.55-57, 2008. 